\title{
Exchange constants for local spin Hamiltonians from tight-binding models
}

\author{
Simon Streib $\odot,{ }_{1}^{1}$ Attila Szilva, ${ }^{1}$ Vladislav Borisov, ${ }^{1}$ Manuel Pereiro, ${ }^{1}$ Anders Bergman, ${ }^{1}$ Erik Sjöqvist $\odot,{ }^{1}$ Anna Delin,${ }^{2,3}$ \\ Mikhail I. Katsnelson $\odot,{ }^{4}$ Olle Eriksson, ${ }^{1,5}$ and Danny Thonig ${ }^{5,1}$ \\ ${ }^{1}$ Department of Physics and Astronomy, Uppsala University, Box 516, SE-75120 Uppsala, Sweden \\ ${ }^{2}$ Department of Applied Physics, School of Engineering Sciences, KTH Royal Institute of Technology, Electrum 229, SE-16440 Kista, Sweden \\ ${ }^{3}$ Swedish e-Science Research Center (SeRC), KTH Royal Institute of Technology, SE-10044 Stockholm, Sweden \\ ${ }^{4}$ Institute for Molecules and Materials, Radboud University, Heyendaalseweg 135, 6525 AJ, Nijmegen, The Netherlands \\ ${ }^{5}$ School of Science and Technology, Örebro University, 70281 Örebro, Sweden
}

(Received 15 March 2021; accepted 1 June 2021; published 10 June 2021)

\begin{abstract}
We consider the mapping of tight-binding electronic structure theory to a local spin Hamiltonian, based on the adiabatic approximation for spin degrees of freedom in itinerant-electron systems. Local spin Hamiltonians are introduced in order to describe the energy landscape of small magnetic fluctuations, locally around a given spin configuration. They are designed for the linear response near a given magnetic state and, in general, are insufficient to capture arbitrarily strong deviations of spin configurations from the equilibrium. In order to achieve this mapping, we include a linear term in the local spin Hamiltonian that together with the usual bilinear exchange tensor, produces an improved accuracy of effective magnetic Weiss fields for noncollinear states. We also provide examples from tight-binding electronic structure theory, where our implementation of the calculation of exchange constants is based on constraining fields that stabilize an out-of-equilibrium spin configuration. We check our formalism by means of numerical calculations for iron dimers and chains.
\end{abstract}

DOI: 10.1103/PhysRevB.103.224413

\section{INTRODUCTION}

The Heisenberg model and generalizations thereof are among the most important paradigms of condensed matter physics and have been very successful in describing the magnetic behavior of both magnetic insulators, for which it was suggested initially, and, with some reservations, metallic magnets. There are several complementary approaches of obtaining the exchange parameters that enter an effective atomistic spin Hamiltonian for a specific material: (a) one can obtain the exchange parameters analytically from a more fundamental electronic Hamiltonian [1-3], (b) one can map the spin Hamiltonian onto total energy calculations for spin spirals [4] and spin-cluster expansions [5-9], or (c) one can use energy variations of the magnetic ground state within firstprinciples approaches such as spin-density functional theory $[10,11]$. While approaches (a) and (b) aim to describe arbitrary spin configurations, approach (c) is explicitly designed to capture small fluctuations around the magnetic ground state. In this paper, we will focus on approach (c), which was pioneered by Liechtenstein, Katsnelson, Antropov, and Gubanov (LKAG) $[10,11]$.

Published by the American Physical Society under the terms of the Creative Commons Attribution 4.0 International license. Further distribution of this work must maintain attribution to the author(s) and the published article's title, journal citation, and DOI. Funded by Bibsam.
In their work, Liechtenstein et al. emphasized that for metals, the Heisenberg Hamiltonian "is applicable only for small spin deviations from the ground state" [11], which implies that terms beyond the bilinear Heisenberg exchange interactions may be required to describe the magnetic behavior for strong deviations from the ground state. This was further confirmed by explicit calculations for the cases of $\mathrm{Fe}$, $\mathrm{Ni}$, and Fe-based magnetic alloys [12]. While the original work by Liechtenstein et al. considered ferromagnetic ground states, extensions of the LKAG formalism to nonequilibrium $[13,14]$ and noncollinear [15-21] states have been considered. However, it was realized that a mapping of noncollinear spin configurations to a Heisenberg model [19] or to a generalized Heisenberg model with a bilinear exchange tensor [17] is, in general, not possible for noncollinear states, apparently requiring the inclusion of higher-order (beyond Heisenberg) exchange contributions [3,9,22]. We propose an alternative solution by including a linear term in a generalized spin model. Linear terms are usually not considered in effective spin Hamiltonians due to arguments connected to the degeneracy of time-reversed states. We argue here that such a linear term can be considered if one is interested only in small fluctuations around a given spin configuration and takes into account that the sign of the linear interaction parameter changes for the spin-reversed configuration. As outlined here, higher-order exchange interactions may not be required in this case, which is in line with the original LKAG approach $[10,11]$.

It is well established that the LKAG formalism is only exact in the long-wavelength regime [23-25]. In an implementation of the adiabatic approximation beyond the long-wavelength limit, the inclusion of constraining fields is 
required [23,26,27]. These constraining fields stabilize noncollinear, out-of-equilibrium spin configurations. We present a formalism for calculating the full bilinear exchange tensor from tight-binding models, which is based on the fact that the effective magnetic field is the negative of the constraining field [26-29]. This follows from the physical picture that the constraining field has to cancel out the effective field acting on a spin. From recent results that the effective field in density functional theory (DFT) contains an additional term besides the constraining field [28], we recover the formula previously derived by Bruno for the isotropic Heisenberg exchange [23], which we extend to the full bilinear exchange tensor. Results for the exchange parameters of nickel from a formalism similar to Ref. [23] have been recently published [30] and show a similar behavior as previous results based on a frozen magnon approach [31], which also takes constraining fields into account. Note that as was shown analytically [25], the Bruno formula corresponds to the extraction of exchange parameters from the inverse static magnetic susceptibility, that is, to the energy of static spiral configurations, whereas LKAG exchanges correspond to the poles of dynamic magnetic susceptibility, that is, to the spin-wave spectrum measured, e.g., by inelastic neutron scattering. In the formal limit of welldefined local magnetic moments where intersite exchange energies are much smaller than on-site Hund exchange splitting, these two expressions coincide.

The paper is organized as follows: in Sec. II, we introduce and define the local spin Hamiltonian and derive an explicit expression for the linear and bilinear terms from the effective magnetic field. In Sec. III, we derive expressions based on the constraining field for the exchange parameters in terms of Green's functions and self-energies. We apply these formulas in Sec. IV to a tight-binding model for iron and present results for iron dimers and chains. Finally, in Sec. V, we summarize our results and provide a broader contextual analysis. In Appendices $\mathrm{A}$ and $\mathrm{B}$, we give additional details on the definition of the effective field and the calculation of Matsubara sums, respectively. In Appendix C, we discuss the symmetry of exchange constants within the different approaches that we consider in this manuscript.

\section{LOCAL SPIN HAMILTONIANS}

We distinguish between two types of spin Hamiltonians: global and local Hamiltonians. With a global Hamiltonian, we denote a Hamiltonian that aims to describe energies of all possible spin configurations [approaches (a) and (b) above], while a local Hamiltonian is designed to describe the energetics of spin configurations in the vicinity of the ground state or, more generally, in the vicinity of a predefined spin configuration [approach (c) above]. Global Hamiltonians are in principle superior, but in practice it may be difficult to obtain the necessary parameters for higher-order exchange interactions if they play a significant role. Also, rigorously speaking, it is not guaranteed that the global Hamiltonian, expressed in terms of spin operators only, exists for itinerant-electron systems at all. Local spin Hamiltonians do not require any spin interactions beyond the bilinear order (for Heisenberg exchange as well as Dzyaloshinskii-Moriya interactions) and the exchange parameters can be directly computed without the need to fit the spin
Hamiltonian to many different spin configurations. However, for a given local spin Hamiltonian, the range of validity, i.e., how small the fluctuations should be, is a priori not known and depends on how significant the higher-order exchange contributions are. Thus, local and global spin Hamiltonians are complementary approaches with distinct advantages and disadvantages. To avoid further misunderstanding, we have to emphasize once more that here we mean "locality" and "globality" of the Hamiltonians in energy and not in real space.

In its most general form, the local spin Hamiltonian we consider in this work is defined as

$$
\mathcal{H}_{s}=-\sum_{i \alpha} C_{i \alpha} e_{i \alpha}-\frac{1}{2} \sum_{i j} \sum_{\alpha \beta} J_{i j}^{\alpha \beta} e_{i \alpha} e_{j \beta},
$$

where $e_{i \alpha}$ is the component $\alpha=x, y, z$ of the magnetic moment unit vector at site $i, J_{i j}^{\alpha \beta}$ is the exchange tensor, and we allow for a linear contribution, $C_{i \alpha}$. Note that in this formulation, the size of the magnetic moment on each site is incorporated in the value of $J_{i j}^{\alpha \beta}$. The linear term in Eq. (2.1) is an important difference between the local and global approaches. In the global approach, a linear term is not allowed because the Hamiltonian (without an external magnetic field) has to be invariant under an inversion of all magnetic moment directions, $e_{i \alpha} \rightarrow-e_{i \alpha}$, due to time-reversal symmetry. For the local approach, the linear term is allowed since only small fluctuations are described and the inversion of all moment directions is beyond this regime. Time-reversal symmetry is recovered by considering that the parameter $C_{i \alpha}$ changes its sign for a time-reversed reference state.

The effective Weiss field of the Hamiltonian (2.1), which is relevant for spin dynamics and for obtaining an equilibrium configuration of the atomic moments, is given by

$$
B_{i \alpha}^{\mathrm{eff}}=-\frac{1}{M_{i}} \frac{\partial \mathcal{H}_{s}}{\partial e_{i \alpha}}=\frac{1}{M_{i}} C_{i \alpha}+\frac{1}{M_{i}} \sum_{k v} J_{i k}^{\alpha \nu} e_{k v},
$$

where $M_{i}$ is the magnetic moment length at site $i$. To specify the parameters of the spin Hamiltonian, we consider the following expansion of the effective field around a given spin configuration $\left\{\mathbf{e}_{i}^{0}\right\}$ to the first order:

$$
B_{i \alpha}^{\mathrm{eff}} \approx B_{i \alpha}^{\mathrm{eff}}\left(\left\{\mathbf{e}_{i}^{0}\right\}\right)+\left.\sum_{j \beta} \frac{\partial B_{i \alpha}^{\mathrm{eff}}}{\partial e_{j \beta}}\right|_{\left\{\mathbf{e}_{i}^{\mathbf{0}}\right\}}\left(e_{j \beta}-e_{j \beta}^{0}\right) .
$$

By comparing Eqs. (2.2) and (2.3), we obtain

$$
\begin{gathered}
J_{i j}^{\alpha \beta}=\left.M_{i} \frac{\partial B_{i \alpha}^{\mathrm{eff}}}{\partial e_{j \beta}}\right|_{\left\{\mathbf{e}_{i}^{0}\right\}}, \\
C_{i \alpha}=M_{i} B_{i \alpha}^{\mathrm{eff}}\left(\left\{\mathbf{e}_{i}^{0}\right\}\right)-\sum_{k v} J_{i k}^{\alpha \nu} e_{k v}^{0} .
\end{gathered}
$$

As required by time-reversal symmetry,

$$
C_{i \alpha}\left(\left\{\mathbf{e}_{i}^{0}\right\}\right)=-C_{i \alpha}\left(\left\{-\mathbf{e}_{i}^{0}\right\}\right) .
$$

If the system under consideration can be exactly described by a bilinear spin Hamiltonian without any higher-order terms, we have

$$
B_{i \alpha}^{\mathrm{eff}}\left(\left\{\mathbf{e}_{i}^{0}\right\}\right)=\frac{1}{M_{i}} \sum_{k v} J_{i k}^{\alpha v} e_{k v}^{0},
$$




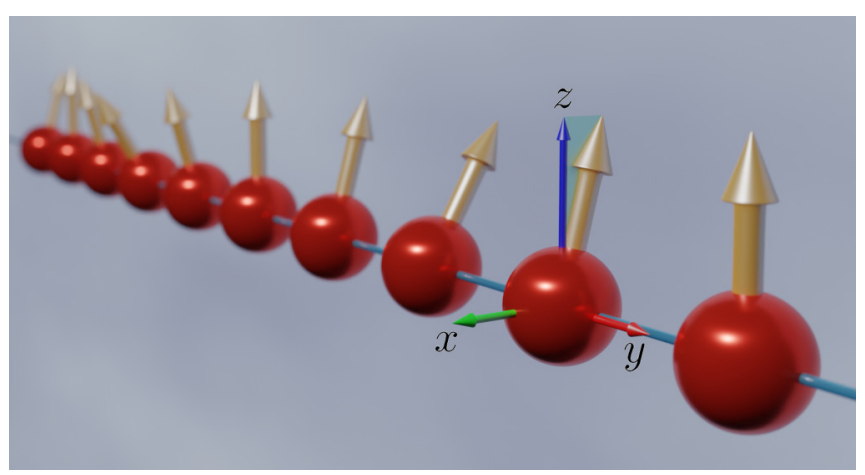

FIG. 1. Illustration of a noncollinear spin chain studied as an example in Sec. II, including the definition of the reference frame.

and the parameter $C_{i \alpha}$ vanishes and is not required. In that case, the local and global spin Hamiltonians are identical. The linear term in the local Hamiltonian, therefore, only plays a role if higher-order exchange interactions are present in the global Hamiltonian.

In the ground state, $\mathbf{B}_{i}^{\text {eff }}=-\nabla_{\mathbf{e}_{i}} E / M_{i}$ vanishes in Eq. (2.5) since the gradient of the energy $E$ has to be zero, and $C_{i \alpha}$ is then determined by the exchange tensor $J_{i j}^{\alpha \beta}$ alone. We note that for the effective magnetic field that drives the precession term of the dynamics of the moment direction $\mathbf{e}_{i}$,

$$
\dot{\mathbf{e}}_{i}=\gamma \mathbf{e}_{i} \times \mathbf{B}_{i}^{\mathrm{eff}},
$$

with $\gamma$ the gyromagnetic ratio, only the component of the effective field that is perpendicular to $\mathbf{e}_{i}$, i.e., $\mathbf{B}_{i \perp}^{\text {eff }}$, contributes due to the cross product.

For a Heisenberg model with a ferromagnetic ground state aligned along the $z$ axis and isotropic exchange tensor,

$$
J_{i j}^{\alpha \beta}=J_{i j} \delta_{\alpha \beta},
$$

we have

$$
\begin{aligned}
& C_{i x}=-\sum_{j} J_{i j}^{x z}=0, \\
& C_{i y}=-\sum_{j} J_{i j}^{y z}=0, \\
& C_{i z}=-\sum_{j} J_{i j}^{z z}=0 .
\end{aligned}
$$

The requirement $J_{i j}^{z z}=0$ follows from the projection to perpendicular effective fields (see Appendix A). Since $C_{i z}$ is the component parallel to the moment direction, it does not contribute to $\mathbf{B}_{i \perp}^{\text {eff }}$ when considering small fluctuations around the ferromagnetic ground state, even if we do not consider the projection to perpendicular fields. Therefore, we recover the established result that no linear terms are required for a ferromagnetic Heisenberg model within the LKAG approach [10,11].

As a simple example, we consider a one-dimensional spin chain (see Fig. 1) with both bilinear and biquadratic nearestneighbor exchange contributions that are selected to result in

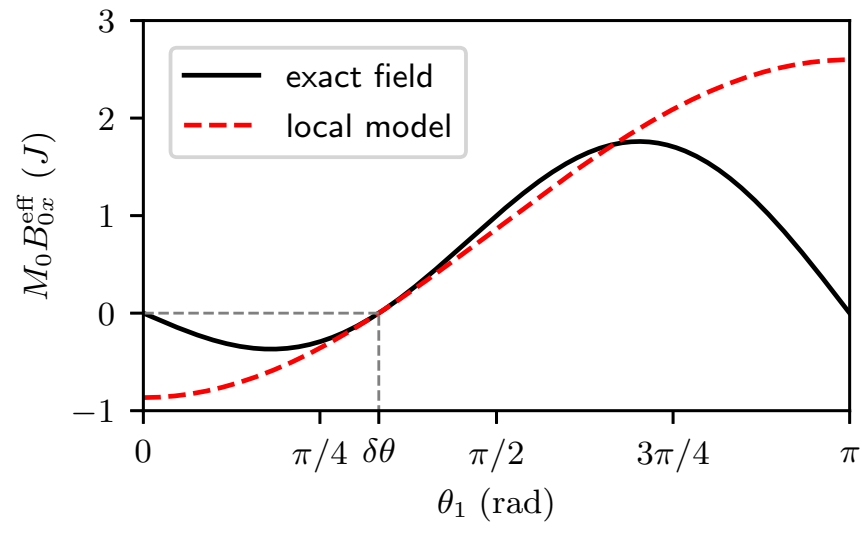

FIG. 2. Comparison of the effective field for the toy model, given by Eq. (2.17), with $J / B=1$, and the corresponding local spin Hamiltonian, given by Eq. (2.2). We consider the effective field acting on the spin at site $i=0$ under rotations of the spin at site $j=1$.

a noncollinear ground state,

$$
\mathcal{H}=-J \sum_{i} \mathbf{e}_{i} \cdot \mathbf{e}_{i+1}+B \sum_{i}\left(\mathbf{e}_{i} \cdot \mathbf{e}_{i+1}\right)^{2},
$$

with $J, B>0$. To obtain the ground state, it is sufficient to consider configurations with

$$
\mathbf{e}_{i} \cdot \mathbf{e}_{i+1}=\cos (\delta \theta) \quad \forall i,
$$

where $\delta \theta$ is the angle between two neighboring spins. The energy is minimized for

$$
\delta \theta= \begin{cases}0, & J / B \geqslant 2 \\ \arccos \left(\frac{J}{2 B}\right), & J / B<2,\end{cases}
$$

i.e., for $J / B<2$, the ground state is noncollinear. For simplicity, we consider here only spin configurations within the $x z$ plane, such that the spin at each site $j$ (specified as an integer) is determined by a single angle $\theta_{j}$, with ground-state value

$$
\theta_{j}=j \delta \theta \text {. }
$$

Figure 2 shows a comparison of the exact effective field, obtained from the gradient of Eq. (2.13),

$$
\begin{aligned}
M_{i} \mathbf{B}_{i}^{\text {eff }}= & -\nabla_{\mathbf{e}_{i}} \mathcal{H} \\
= & J\left(\mathbf{e}_{i+1}+\mathbf{e}_{i-1}\right) \\
& -2 B\left(\mathbf{e}_{i+1}\left[\mathbf{e}_{i} \cdot \mathbf{e}_{i+1}\right]+\mathbf{e}_{i-1}\left[\mathbf{e}_{i} \cdot \mathbf{e}_{i-1}\right]\right),
\end{aligned}
$$

for $J / B=1$ (for which $\delta \theta=\pi / 3$ ) and the effective field obtained from the local spin Hamiltonian, given by the last part of Eq. (2.2). The results are obtained for the spin at site $i=0$ under rotations of the spin $j=1$ with angle $\theta_{1}$, while all other spins are in their noncollinear ground-state configuration. The relevant ground-state effective exchange parameters are

$$
\begin{gathered}
J_{0,1}^{x x}=J_{0,-1}^{x x}=J-2 B \cos (\delta \theta), \\
J_{0,1}^{x z}=-J_{0,-1}^{x z}=-2 B \sin (\delta \theta) .
\end{gathered}
$$

The effective field vanishes in the ground state, $\theta_{1}=\delta \theta$, as expected. The linear term $C_{0 x}$ vanishes in this example due to a mirror symmetry with respect to the spins at sites -1 and +1 with $\theta_{+1}=-\theta_{-1}$. As the figure shows, the local 
spin Hamiltonian provides an excellent approximation of the effective field for small fluctuations around the ground state. The result suggests that a local spin Hamiltonian is sufficient for the calculation of, e.g., spin-wave spectra since they only depend on energy variations near the ground state.

\section{EXCHANGE CONSTANTS}

We consider here the derivation of the exchange tensor $J_{i j}^{\alpha \beta}$ of the local spin Hamiltonian (2.1) based on a tight-binding formalism. The standard approach is to consider variations of the electronic energy $[10,11]$, which may be used to obtain the exchange parameters,

$$
J_{i j}^{\alpha \beta}=-\frac{\partial^{2}\langle\hat{\mathcal{H}}\rangle}{\partial e_{i \alpha} \partial e_{j \beta}} .
$$

From the point of view of a global spin Hamiltonian that may contain higher-order exchange contributions, these exchange parameters $J_{i j}^{\alpha \beta}$ are not just the bilinear exchange parameters of such a global Hamiltonian but also take higherorder exchange contributions into account [see Eqs. (2.18) and (2.19)], which causes a configuration dependence of $J_{i j}^{\alpha \beta}[12,22]$.

Based on the result that the effective magnetic field, when not considering DFT calculations, can just be obtained from the constraining field [28],

$$
B_{i \alpha}^{\text {eff }}=-\frac{1}{M_{i}} \frac{\partial\langle\hat{\mathcal{H}}\rangle}{\partial e_{i \alpha}}=-B_{i \alpha}^{\text {con }},
$$

here we are taking the alternative approach of calculating the exchange parameters from the constraining field,

$$
J_{i j}^{\alpha \beta}=-M_{i} \frac{\partial B_{i \alpha}^{\text {con }}}{\partial e_{j \beta}},
$$

which is equivalent to Eq. (3.1) if we assume $M_{i}=$ const. The constraining field $\mathbf{B}_{i}^{\text {con }}$ is perpendicular to the moment direction $\mathbf{e}_{i}$ and is required to stabilize nonequilibrium spin configurations within the adiabatic approximation [26-28,32]. It is added here to the full electronic tight-binding Hamiltonian $\hat{\mathcal{H}}_{\mathrm{tb}}$,

$$
\hat{\mathcal{H}}=\hat{\mathcal{H}}_{\mathrm{tb}}+\hat{\mathcal{H}}_{\mathrm{con}},
$$

with

$$
\hat{\mathcal{H}}_{\mathrm{con}}=-\sum_{i} \gamma \hat{\mathbf{S}}_{i} \cdot \mathbf{B}_{i}^{\mathrm{con}},
$$

where $\hat{\mathbf{S}}_{i}$ is the spin operator at lattice site $i$.

The tight-binding Hamiltonian consists of a hopping term $\hat{\mathcal{H}}_{0}$ and an interaction term $\hat{\mathcal{H}}_{\text {int }}$,

$$
\hat{\mathcal{H}}_{\text {tb }}=\hat{\mathcal{H}}_{0}+\hat{\mathcal{H}}_{\text {int }} \text {. }
$$

The hopping term is, in second quantization, given by

$$
\hat{\mathcal{H}}_{0}=\sum_{i \ell, j \ell^{\prime}, \sigma} t_{i \ell, j \ell^{\prime}} \hat{c}_{i \ell \sigma}^{\dagger} \hat{c}_{j \ell^{\prime} \sigma}
$$

which describes the hopping of an electron from state $j \ell^{\prime} \sigma$ to $i \ell \sigma$ with hopping amplitude $t_{i \ell, j \ell^{\prime}}$ and creation and annihilation operators $\hat{c}_{i \ell \sigma}^{\dagger}$ and $\hat{c}_{j \ell^{\prime} \sigma}$. The index $i \ell \sigma$ indicates the lattice site, orbit, and spin, respectively. The hopping amplitudes are assumed to be constant parameters that do not depend on the magnetic state of the system, which is a consequence of expressing the tight-binding Hamiltonian in Eq. (3.7) in a global spin basis (defined along a common $z$ axis). The form of the hopping part of the Hamiltonian in Eq. (3.7) is that of a matrix which is block diagonal in spin space, where each block has, e.g., for $d$ states, dimension $5 \times 5$. Furthermore, the interaction term $\hat{\mathcal{H}}_{\text {int }}$ includes the Coulomb and spin-orbit interactions, where the former interaction is responsible for spin pairing and the possibility of forming a finite magnetic moment on each lattice site. Within a mean-field approximation, these interactions could, in principle, be included as a spin-dependent hopping term, but we choose to keep the separation between the spinindependent hopping in $\hat{\mathcal{H}}_{0}$ and the interaction term $\hat{\mathcal{H}}_{\text {int }}$, such that $\hat{\mathcal{H}}_{0}$ is independent of the magnetic state. Within this formalism, a spin-dependent hopping contribution could, in principle, still be included in $\hat{\mathcal{H}}_{\text {int }}$ and in the corresponding self-energy $\Sigma$.

\section{A. Exchange from constraining field}

To obtain the exchange tensor $J_{i j}^{\alpha \beta}$, we have to calculate the derivative of the constraining field. Our starting point is to calculate the change of the magnetic moment component $M_{j \beta}$ under a change of the prescribed directions $\left\{\mathbf{e}_{i}\right\}$ [23], from which we obtain the set of equations

$$
\frac{\partial M_{j \beta}}{\partial e_{i \alpha}}=\frac{\partial\left(M_{j} e_{j \beta}\right)}{\partial e_{i \alpha}} \approx M_{j} \frac{\partial e_{j \beta}}{\partial e_{i \alpha}}=M_{i} \delta_{\alpha \beta} \delta_{i j},
$$

where we assume a constant magnetic moment length $M_{j}$. This approximation is valid in the magnetic ground state [11]. Keeping the moment length fixed introduces to the constraining field a spurious contribution that is parallel to the moment direction, which has, however, no relevance for the spin dynamics, given by Eq. (2.8), and can be projected out (see Appendix A).

The derivative of $M_{j \beta}$ can be obtained by expressing the expectation value via Matsubara Green's functions,

$$
\begin{gathered}
M_{j \beta}=\frac{\hbar \gamma}{2} \sum_{\ell} \sum_{\sigma \sigma^{\prime}} \sigma_{\sigma \sigma^{\prime}}^{\beta} \int_{\omega}[\mathcal{G}(i \omega)]_{j \ell \sigma^{\prime}, j \ell \sigma} \\
=\frac{\hbar \gamma}{2} \int_{\omega} \operatorname{Tr}\left\{\sigma_{j}^{\beta} \mathcal{G}(i \omega)\right\},
\end{gathered}
$$

with matrix elements

$$
\left[\sigma_{k}^{\beta}\right]_{i \ell \sigma, j \ell^{\prime} \sigma^{\prime}}=\sigma_{\sigma \sigma^{\prime}}^{\beta} \delta_{i k} \delta_{j k} \delta_{\ell \ell^{\prime}},
$$

where $\sigma_{\sigma \sigma^{\prime}}$ is the Pauli matrix vector. We use the following shorthand notation for the Matsubara sums,

$$
\int_{\omega} \equiv k_{B} T \sum_{i \omega} e^{i \omega 0^{+}},
$$

with Boltzmann constant $k_{B}$ and temperature $T$. We include the required convergence factor $e^{i \omega 0^{+}}$for the correct time ordering of the operators.

From the inverse matrix derivative rule,

$$
\partial \mathcal{G}=-\mathcal{G}\left(\partial \mathcal{G}^{-1}\right) \mathcal{G},
$$


together with the Dyson equation,

$$
\mathcal{G}^{-1}=\mathcal{G}_{0}^{-1}-\Sigma,
$$

we obtain

$$
\frac{\partial M_{j \beta}}{\partial e_{i \alpha}}=\frac{\hbar \gamma}{2} \int_{\omega} \operatorname{Tr}\left\{\sigma_{j}^{\beta} \mathcal{G}(i \omega)\left[\frac{\partial \mathcal{H}_{\mathrm{con}}}{\partial e_{i \alpha}}+\frac{\partial \Sigma(i \omega)}{\partial e_{i \alpha}}\right] \mathcal{G}(i \omega)\right\},
$$

where we assume a constant chemical potential $\mu$ (see Appendix B for the definition of the Green's functions). Here we have used that the noninteracting Green's function $\mathcal{G}_{0}$ only depends on the moment directions via the constraining field contribution (which we include in $\mathcal{G}_{0}$ ),

$$
\frac{\partial \mathcal{G}_{0}^{-1}}{\partial e_{i \alpha}}=-\frac{\partial \mathcal{H}_{\mathrm{con}}}{\partial e_{i \alpha}} .
$$

All other contributions that depend on the moment directions are, by definition, included in the self-energy $\Sigma$, which takes correlation effects from $\hat{\mathcal{H}}_{\text {int }}$ into account. From the matrix of the constraining part of the Hamiltonian,

$$
\mathcal{H}_{\text {con }}=-\frac{\hbar \gamma}{2} \sum_{k v} \sigma_{k}^{v} B_{k v}^{\mathrm{con}},
$$

we obtain the corresponding derivative,

$$
\frac{\partial \mathcal{H}_{\mathrm{con}}}{\partial e_{i \alpha}}=-\frac{\hbar \gamma}{2} \sum_{k \nu} \sigma_{k}^{v} \frac{\partial B_{k \nu}^{\mathrm{con}}}{\partial \boldsymbol{e}_{i \alpha}} .
$$

We can now write

$$
\frac{\partial M_{j \beta}}{\partial e_{i \alpha}}=K_{j i}^{\beta \alpha}+\sum_{k v} X_{j k}^{\beta \nu} \frac{\partial B_{k v}^{\text {con }}}{\partial e_{i \alpha}},
$$

with

$$
\begin{gathered}
K_{i j}^{\alpha \beta}=\frac{\hbar \gamma}{2} \int_{\omega} \operatorname{Tr}\left\{\sigma_{i}^{\alpha} \mathcal{G}(i \omega)\left[\frac{\partial \Sigma(i \omega)}{\partial e_{j \beta}}\right] \mathcal{G}(i \omega)\right\}, \\
X_{i j}^{\alpha \beta}=-\frac{\hbar^{2} \gamma^{2}}{4} \int_{\omega} \operatorname{Tr}\left\{\sigma_{i}^{\alpha} \mathcal{G}(i \omega) \sigma_{j}^{\beta} \mathcal{G}(i \omega)\right\} .
\end{gathered}
$$

Inserting Eq. (3.19) into Eq. (3.8) gives

$$
\sum_{k \nu} X_{j k}^{\beta v} \frac{\partial B_{k \nu}^{\text {con }}}{\partial e_{i \alpha}}=M_{i} \delta_{\alpha \beta} \delta_{i j}-K_{j i}^{\beta \alpha}
$$

By multiplying with $X^{-1}$, we finally obtain

$$
J_{i j}^{\alpha \beta}=-M_{i} \sum_{k \nu}\left[X^{-1}\right]_{i k}^{\alpha \nu}\left(M_{j} \delta_{\beta \nu} \delta_{j k}-K_{k j}^{\nu \beta}\right) .
$$

If the derivative $\partial \Sigma / \partial e_{i \alpha}$ is not easily accessible but $\partial \Sigma / \partial B_{i \alpha}^{\text {con }}$ is, then we can take a slightly different approach. We write

$$
\frac{\partial M_{j \beta}}{\partial e_{i \alpha}}=\sum_{k \nu} \tilde{X}_{j k}^{\beta \nu} \frac{\partial B_{k v}^{\text {con }}}{\partial e_{i \alpha}},
$$

with

$$
\tilde{X}_{i j}^{\alpha \beta}=-\frac{\hbar^{2} \gamma^{2}}{4} \int_{\omega} \operatorname{Tr}\left\{\sigma_{i}^{\alpha} \mathcal{G}(i \omega)\left[\sigma_{j}^{\beta}-\frac{2}{\hbar \gamma} \frac{\partial \Sigma(i \omega)}{\partial B_{j \beta}^{\text {con }}}\right] \mathcal{G}(i \omega)\right\} .
$$

We obtain then the alternative but equivalent result,

$$
J_{i j}^{\alpha \beta}=-M_{i} M_{j}\left[\tilde{X}^{-1}\right]_{i j}^{\alpha \beta} .
$$

This reformulation is useful when the self-energy is obtained from a diagrammatic expansion in terms of noninteracting Green's functions, where the derivative of the self-energy with respect to the constraining field can be performed analytically.

\section{B. DFT-like correction term}

In DFT calculations with constraining fields, the effective magnetic field is given by the energy gradient which, in this case, is not identical to the negative of the constraining field [28],

$$
\mathbf{B}_{i}^{\text {eff }}=-\frac{1}{M_{i}} \nabla_{\mathbf{e}_{i}} E=-\mathbf{B}_{i}^{\text {con }}-\frac{1}{M_{i}}\left\langle\nabla_{\mathbf{e}_{i}}^{*} \hat{\mathcal{H}}_{\mathrm{KS}}\right\rangle,
$$

where $\hat{\mathcal{H}}_{\mathrm{KS}}$ is the auxiliary Kohn-Sham Hamiltonian [33] and $\nabla_{\mathbf{e}_{i}}^{*}$ denotes the derivative with constant electron densities and moment lengths [11]. Although in the present investigation we are considering tight-binding models, we may need to take this correction term into account if the tight-binding model has been fitted to Kohn-Sham band structures and the DFT formalism has to be applied for consistency. The self-consistent exchange constants are then obtained from a derivative of Eq. (3.27),

$$
J_{\mathrm{sc}, i j}^{\alpha \beta}=-M_{i} \frac{\partial B_{i \alpha}^{\mathrm{con}}}{\partial e_{j \beta}}-\frac{\partial}{\partial e_{j \beta}}\left\langle\frac{\partial^{*}}{\partial e_{i \alpha}} \hat{\mathcal{H}}_{\mathrm{KS}}\right\rangle .
$$

We assume now that we have a tight-binding Hamiltonian that reproduces the band structure of $\hat{\mathcal{H}}_{\mathrm{KS}}$, where the exchange splitting is parameterized via the following Stoner term [34-36]:

$$
\hat{\mathcal{H}}_{\mathrm{St}}=\sum_{i \ell \ell^{\prime}} \frac{I_{\ell \ell^{\prime}}}{\hbar \mu_{B}} M_{i \ell} \mathbf{e}_{i} \cdot \hat{\mathbf{S}}_{i \ell^{\prime}},
$$

where $M_{i \ell}$ is the magnetic moment length and $\hat{\mathbf{S}}_{i \ell}$ the spin operator associated with the orbital $\ell$ at site $i$ with Stoner parameter $I_{\ell \ell^{\prime}}$. From

$$
\left\langle\frac{\partial^{*}}{\partial e_{i \alpha}} \hat{\mathcal{H}}_{\mathrm{St}}\right\rangle=\sum_{\ell \ell^{\prime}} \frac{I_{\ell \ell^{\prime}} M_{i \ell}}{\hbar \gamma \mu_{B}} M_{i \ell^{\prime} \alpha},
$$

we obtain

$$
\frac{\partial}{\partial e_{j \beta}}\left\langle\frac{\partial^{*}}{\partial e_{i \alpha}} \hat{\mathcal{H}}_{\mathrm{St}}\right\rangle=\sum_{\ell \ell^{\prime}} \frac{I_{\ell \ell^{\prime}} M_{i \ell}}{\hbar \gamma \mu_{B}} \frac{\partial M_{i \ell^{\prime} \alpha}}{\partial e_{j \beta}},
$$

where we assume that both $I_{\ell \ell^{\prime}}$ and $M_{i \ell}$ are constant. Next, analogous to Eq. (3.19), we derive an expression for the derivative of the orbital resolved magnetic moments,

$$
\frac{\partial M_{j \ell \beta}}{\partial e_{i \alpha}}=K_{j i}^{\beta \alpha}(\ell)+\sum_{k \nu} X_{j k}^{\beta \nu}(\ell) \frac{\partial B_{k \nu}^{\text {con }}}{\partial e_{i \alpha}},
$$

where $(\ell)$ denotes that we restrict the trace in the calculation of the matrices to a single orbital with index $\ell$. Combining Eqs. (3.32), (3.31), and (3.28), we obtain

$$
J_{\mathrm{sc}, i j}^{\alpha \beta}=J_{i j}^{\alpha \beta}+J_{0, i j}^{\alpha \beta}+\sum_{k \nu} \frac{1}{M_{k}} K_{k i}^{\nu \alpha} J_{k j}^{\nu \beta},
$$


where

$$
J_{0, i j}^{\alpha \beta}=-\int_{\omega} \operatorname{Tr}\left\{\frac{\partial \Sigma}{\partial e_{i \alpha}} \mathcal{G}(i \omega) \frac{\partial \Sigma}{\partial e_{j \beta}} \mathcal{G}(i \omega)\right\}
$$

is the contribution that we would get without the constraining field and $J_{i j}^{\alpha \beta}$ is the pure constraining field contribution derived in Sec. III A. Here, the self-energy is given by the Stoner term,

$$
\Sigma=\mathcal{H}_{\text {St }},
$$

which is, by definition, included in the self-energy due to the dependence of $\mathcal{H}_{\mathrm{St}}$ on the moment directions.

The structure of Eq. (3.33) corresponds to the DFT results by Bruno [23] for isotropic Heisenberg exchange parameters $J_{i j}=J_{i j}^{x x}=J_{i j}^{y y}$ and an equation equivalent to Eq. (3.34) has been previously derived by Katsnelson and Lichtenstein [37], again only for Heisenberg parameters $J_{i j}$ (see, also, Ref. [38]). Exchange parameters calculated without constraining fields have been shown to give the exact spin-wave energies in the long-wavelength limit [23-25], i.e., for the calculation of the exchange stiffness constant, it is not necessary to consider constraining fields.

\section{NUMERICAL RESULTS}

We have three different equations available for the calculation of exchange parameters: Eq. (3.23), which is based on the constraining field; Eq. (3.33), which is valid for DFT calculations or parametrized calculations mimicking DFT results (as employed here) that include the constraining fields; and Eq. (3.34), which is obtained without constraining fields. We have implemented these three equations within the CAHMD package [39] and applied them to a mean-field tight-binding model for iron with a ferromagnetic ground state. We compare the results of each approach for iron dimers and iron chains with a lattice constant of $2.86 \AA$. The Slater-Koster parameters [40] of the tight-binding model are taken from Ref. [41], we do not include spin-orbit coupling, and we use the Stoner term (3.29); see Ref. [28] for details. For the numerical evaluation of the exchange constants, we use a finite temperature parameter, e.g., $T=1 \mathrm{~K}$, to avoid divergences in the derivative of the Fermi function; see Appendix B. Since we do not take finite temperature effects on the electronic structure and lattice vibrations [42] into account, we will consider only the zero-temperature limit.

\section{A. Fe dimer}

We first compare, in Fig. 3, for an iron dimer, the calculated exchange constant $J_{12}^{x x}$ from Eq. (3.23) with the result obtained by numerical differentiation of the constraining field,

$$
J_{12}^{x x}=-M_{1} \frac{\partial B_{1 x}^{\text {con }}}{\partial e_{2 x}} .
$$

We keep the first moment aligned along the $z$ axis and rotate the second moment by an angle $\theta$ in the $x z$ plane. The rotation is performed by adjusting the moment direction in the Stoner term (3.29) and applying the required constraining field to stabilize the configuration. In the limit $\theta \rightarrow 0$ (the ferromagnetic ground state), the agreement is exact, while for $\theta>0$, there is a small difference which is due to the dependencies

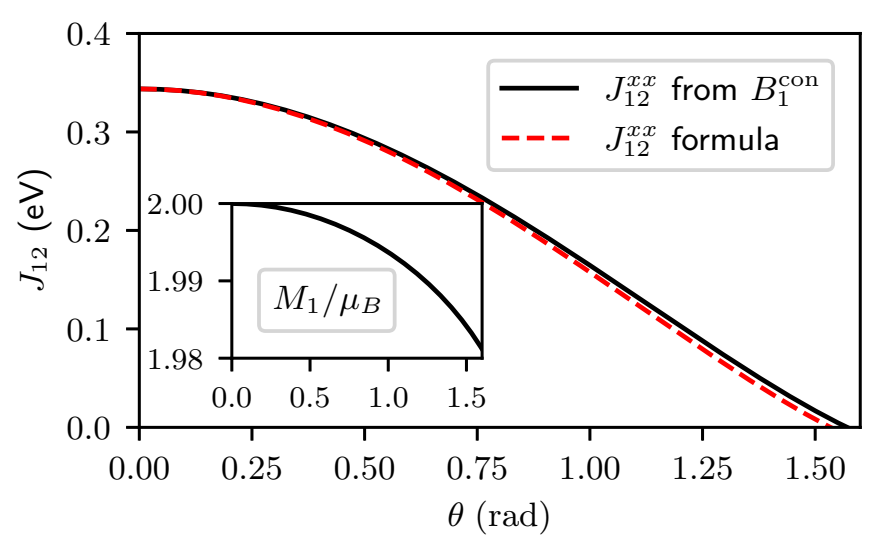

FIG. 3. Comparison of the exchange parameter $J_{12}^{x x}$ obtained by numerical differentiation of the constraining field, given by Eq. (4.1), and the one obtained from Eq. (3.23) as a function of the angle $\theta$ between the two magnetic moments of an iron dimer. The inset shows the magnetic moment length $M_{1}\left(=M_{2}\right)$.

of the magnetic moments and the chemical potential on the spin configuration that are both not taken into account in our derivation of the exchange constants. The magnetic moments, with $M_{1}=M_{2}$, vary by about $1 \%$ and the chemical potential by about $2 \%$ in the range $\theta=0$ to $\pi / 2$; see Figs. 3 and 8 in Appendix B.

Next, we consider the exchange tensor $J_{12}^{\alpha \beta}$ as a function of the angle $\theta$ between the two moments. After projection to perpendicular fields according to Eqs. (A6) and (A7) in Appendix A, which removes the spurious contribution mentioned after Eq. (3.8), only the components $x x, y y$, and $x z$ are finite and are shown in Fig. 4. In the ferromagnetic ground state, $\theta=0$, we have $J_{x x}=J_{y y}$ and $J_{x z}=0$, indicating a Heisenberg-like local spin Hamiltonian. However, for $\theta>0$, $J_{x x} \neq J_{y y}$ and $J_{x z} \neq 0$, implying a non-Heisenberg-like behavior, which requires the inclusion of the linear term $C_{i \alpha}$ in the local spin Hamiltonian.

We calculate the exchange parameters from Eq. (3.23) for two specific angles $(\theta=0$ and $\theta=1)$ as examples and apply them to the local spin Hamiltonian, given by Eq. (2.1). In Fig. 5, we compare the effective field for these two cases

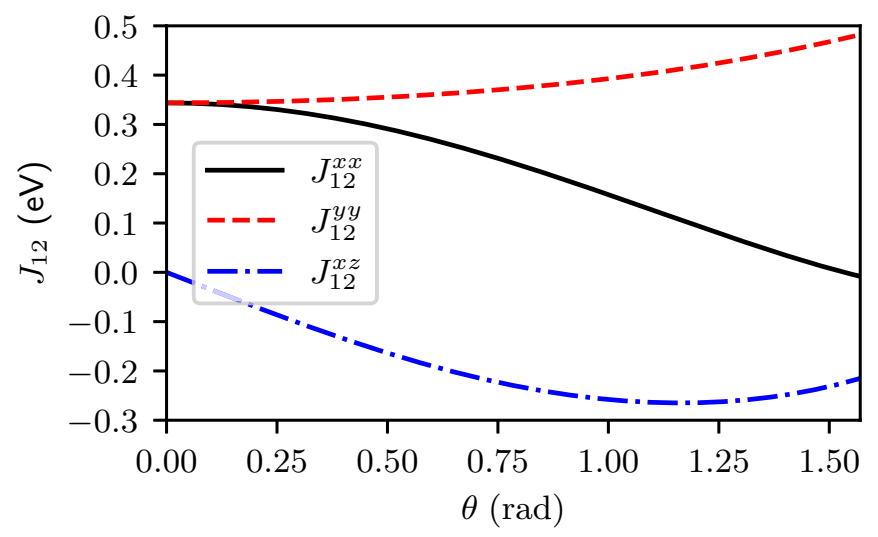

FIG. 4. Components of the exchange tensor $J_{12}^{\alpha \beta}$ obtained from Eq. (3.23) as a function of the angle $\theta$ between the two magnetic moments of an iron dimer. 


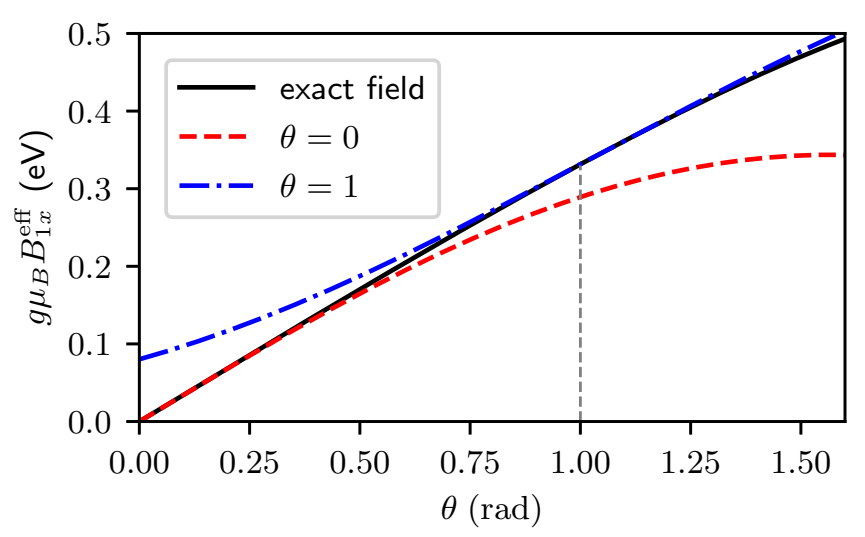

FIG. 5. Comparison of the effective field obtained from the constraining field with the field from the local spin Hamiltonian, given by Eq. (2.1), with parameters calculated with Eq. (3.23) for $\theta=0$ and $\theta=1$, as a function of the angle $\theta$ between the two magnetic moments of an iron dimer.

with the effective field given by the negative of the constraining field. For $\theta=0$, the local spin Hamiltonian is a simple Heisenberg model with a single exchange parameter $J=J^{x x}=J^{y y}$, while for $\theta=1$, the full exchange tensor $J_{i j}^{\alpha \beta}$ and the linear term $C_{i \alpha}$ have to be taken into account with $C_{1 x}=0.3315 \mathrm{eV}$. The linear term includes a contribution from the effective field as defined in Eq. (2.5). In both cases, the local spin Hamiltonian correctly describes small deviations around the reference spin configurations, $\theta=0$ and $\theta=1$. The deviations of the exact field from the Heisenberg model confirms that beyond-Heisenberg contributions are present in the underlying system, which is consistent with the configuration-dependent exchange parameters in Fig. 4.

We compare, for the ferromagnetic ground state $(\theta=0)$, the Heisenberg exchange constants from Eqs. (3.23), (3.34), and (3.33),

$$
\begin{gathered}
J=0.3437 \mathrm{eV}, \\
J_{0}=0.2575 \mathrm{eV}, \\
J_{\text {sc }}=0.3485 \mathrm{eV} .
\end{gathered}
$$

While the correction term changes $J_{\text {sc }}$ in comparison to $J$ by only a small amount, the difference to $J_{0}$, which is obtained without the inclusion of constraining fields, is more significant. For comparison, a recent tight-binding calculation with constraining fields obtained $J=0.616 \mathrm{eV}$ for an iron dimer with a lattice constant of $2 \AA$ [29], where the smaller lattice constant causes a stronger exchange coupling than in our case with lattice constant $2.86 \AA$. In DFT calculations, similar deviations of the nearest-neighbor exchange with and without constraining fields have been observed for bulk bcc Fe and fcc $\mathrm{Ni}$, while the energies of long-wavelength spin waves are unaffected by constraining fields [30,31].

Although the difference between $J$ and $J_{\text {sc }}$ is very small, the difference between the effective fields with and without the DFT-like correction term can become significant in noncollinear states [28]. This can be understood by considering that the exchange parameters give the derivative of

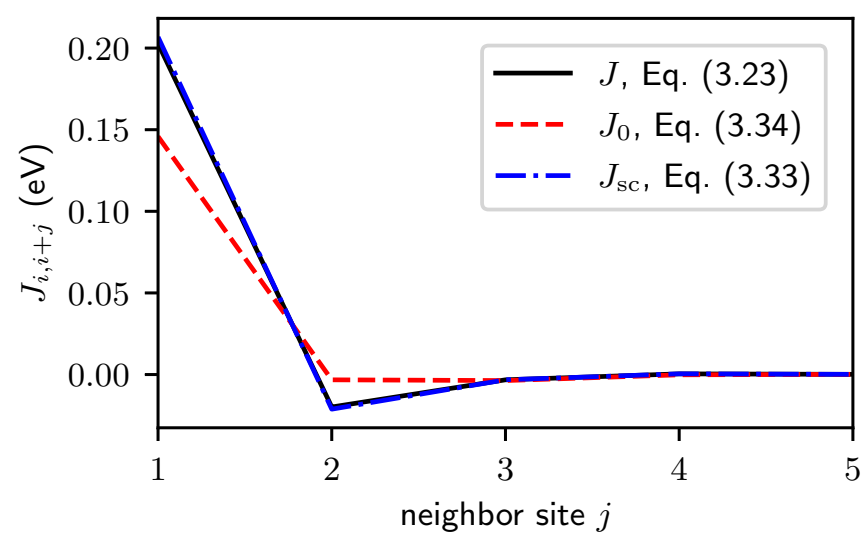

FIG. 6. Exchange parameters $J_{i, i+j}$ between sites $i$ and $i+j$ obtained from Eqs. (3.23), (3.34), and (3.33) for an iron chain of 50 sites in the ferromagnetic ground-state configuration with periodic boundary conditions.

the effective field and a small difference in the derivative can change the effective field significantly for strongly noncollinear states.

While the exchange constants $J_{0, i j}^{\alpha \beta}$ and $J_{\mathrm{sc}, i j}^{\alpha \beta}$ are always symmetric with respect to the interchange $i \alpha \stackrel{\leftrightarrow}{\leftrightarrow} \beta$, we find a small asymmetry for $J_{i j}^{\alpha \beta}$ in noncollinear states $(\theta \neq 0)$ in our numerical calculations, which we discuss in Appendix $\mathrm{C}$.

\section{B. Fe chain}

In Fig. 6, we show the Heisenberg exchange parameters $J_{i j}$ for an iron chain in its ferromagnetic ground state, where again the formulas based on the constraining field and the parameterized DFT-like formalism give similar results, given by Eqs. (3.23) and (3.33), while the results without constraining field, given by Eq. (3.34), differ significantly. In Fig. 7, we compare the results for the nearest-neighbor exchange $J$, given by Eq. (3.23), in a finite iron chain of 50 spins with and without periodic boundary conditions. As expected, in the case with periodic boundary conditions, the nearest-neighbor exchange is completely uniform for all sites, while for the

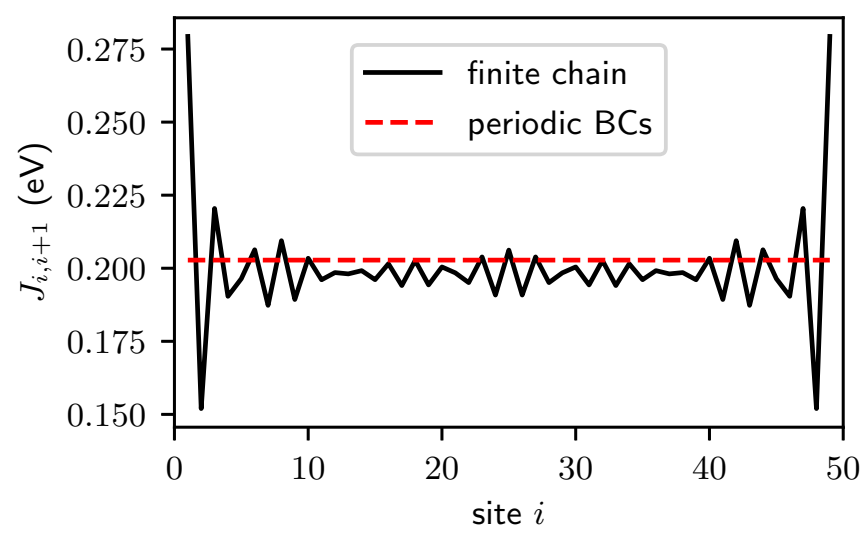

FIG. 7. Nearest-neighbor exchange parameters $J_{i, i+1}$ obtained from Eq. (3.23) for a finite iron chain of 50 sites in the ferromagnetic ground-state configuration in comparison to a chain with periodic boundary conditions. 
case without periodic boundary conditions, there are strong variations near the boundaries of the chain and the deviations become smaller near the center. This reflects Friedel oscillations in the magnetic profile, induced by the abrupt change of the effective potential and hopping parameter at the edges. Such variations of the exchange constants near the boundary of a magnet could be important for a proper description of surface and edge spin waves and topological magnons in twoand three-dimensional magnets [43].

\section{SUMMARY}

We have derived the mapping of tight-binding electronic structure theory to local spin Hamiltonians. We show that in order to capture effects beyond bilinear Heisenberg exchange, the inclusion of a linear term to the spin Hamiltonian improves the accuracy of calculating, e.g., a local Weiss field. Linear contributions are usually not considered due to arguments based on the energy of time-reversed states. We argue here that this is not a problem for local spin Hamiltonians, which are designed to describe the energetics of spin fluctuations around a given spin configuration, in particular by consideration of configuration-dependent parameters. Local spin Hamiltonians are shown to be useful for the calculation of spin-wave spectra and spin dynamics simulations near to the ground-state configuration.

We also provide explicit formulas for the exchange constants based on a derivation from the effective field for tight-binding models with and without constraining fields. If we consider the effective field that is required for DFT calculations [28], we recover previous results [23,37], demonstrating the consistency of our approach. We apply the derived formulas to a tight-binding model for iron dimers and chains, and find good agreement with the exchange constant derived by numerical differentiation of the constraining field. The numerical tight-binding electronic structure theory calculations in Sec. IV are based on a formulation where spin functions are defined along a global quantization axis. In this formulation, the tight-binding parameters (that typically are parametrized to reproduce static electronic structures obtained from $a b$ initio theory) are fixed and independent of the magnetic configuration. This implies that the configuration dependence of the kinetic energy does not enter the equations of exchange interactions or the local Weiss field. A description that relies on a local quantization axis, for which spin functions are defined on each atomic site, would release this constraint and represents an obvious extension of this work.

The local spin Hamiltonian, together with the exchange constant formulas, is demonstrated to correctly describe the effective field near a given spin configuration. We find, however, that for larger deviations from a given spin configuration from which the exchange parameters were calculated, there can be a pronounced configuration dependence of the parameters. This fact implies that beyond-Heisenberg contributions are required and are effectively taken into account.

While for the description of arbitrary spin configurations a global spin Hamiltonian is required, we expect that the local approach described here will find applications to characterize spin waves and spin fluctuations for magnets with noncollinear ground states. A consistent extension of the
LKAG approach to these noncollinear states would not be possible without the linear term in the Hamiltonian or by inclusion of higher-order terms [17]. The exchange constant formulas that we have derived for tight-binding models will be useful both for model Hamiltonians and for ab initio electronic structure calculations.

\section{ACKNOWLEDGMENTS}

We thank Pavel Bessarab, Ksenia Vodenkova, and Lars Nordström for insightful discussions. The authors acknowledge financial support from the Knut and Alice Wallenberg Foundation through Grant No. 2018.0060. O.E. also acknowledges support of eSSENCE, the Swedish Research Council (VR), the Foundation for Strategic Research (SSF), and ERC synergy Grant No. 854843-FASTCORR. D.T. acknowledges support from the Swedish Research Council (VR) through Grant No. 2019-03666. A.D. acknowledges support from the Swedish Research Council (VR) through Grants No. VR 2015-04608, No. VR 2016-05980, and No. VR 2019-05304. The work of M.I.K. is supported by ERC synergy Grant No. 854843-FASTCORR. The computations were enabled by resources provided by the Swedish National Infrastructure for Computing (SNIC) at Chalmers Center for Computational Science and Engineering (C3SE), High Performance Computing Center North (HPCN), and the National Supercomputer Center (NSC), partially funded by the Swedish Research Council through Grant Agreement No. 2016-07213.

\section{APPENDIX A: PROJECTION TO PERPENDICULAR FIELDS}

If we consider a spin Hamiltonian

$$
\mathcal{H}_{s}=-\sum_{i \alpha} \tilde{C}_{i \alpha} e_{i \alpha}-\frac{1}{2} \sum_{i j} \sum_{\alpha \beta} \tilde{J}_{i j}^{\alpha \beta} e_{i \alpha} e_{j \beta},
$$

that does not necessarily result in effective fields that are purely perpendicular to the magnetic moment directions,

$$
B_{i \alpha}^{\mathrm{eff}}=\frac{1}{M_{i}} \tilde{C}_{i \alpha}+\frac{1}{M_{i}} \sum_{k v} \tilde{J}_{i k}^{\alpha v} e_{k v}
$$

then we can project out the parallel component,

$$
\mathbf{B}_{i \perp}^{\text {eff }}=\mathbf{B}_{i}^{\text {eff }}-\mathbf{e}_{i}\left(\mathbf{B}_{i}^{\text {eff }} \cdot \mathbf{e}_{i}\right) .
$$

From this projection, we obtain parameters that produce purely perpendicular fields,

$$
\begin{gathered}
J_{i j}^{\alpha \beta}=\tilde{J}_{i j}^{\alpha \beta}-\sum_{\nu} \tilde{J}_{i j}^{\nu \beta} e_{i v} e_{i \alpha}, \\
\mathbf{C}_{i}=\tilde{\mathbf{C}}_{i}-\mathbf{e}_{i}\left(\tilde{\mathbf{C}}_{i} \cdot \mathbf{e}_{i}\right) .
\end{gathered}
$$

For a state with $\mathbf{e}_{i}=\hat{\mathbf{e}}_{z}$, we have

$$
\begin{gathered}
J_{i j}^{\alpha \beta}=\tilde{J}_{i j}^{\alpha \beta}(\text { for } \alpha \neq z), \\
J_{i j}^{z \beta}=0, \\
C_{i z}=0 .
\end{gathered}
$$

This projection to perpendicular fields can break the symmetry $J_{i j}^{\alpha \beta}=J_{j i}^{\beta \alpha}$ and is not required for practical calculations 


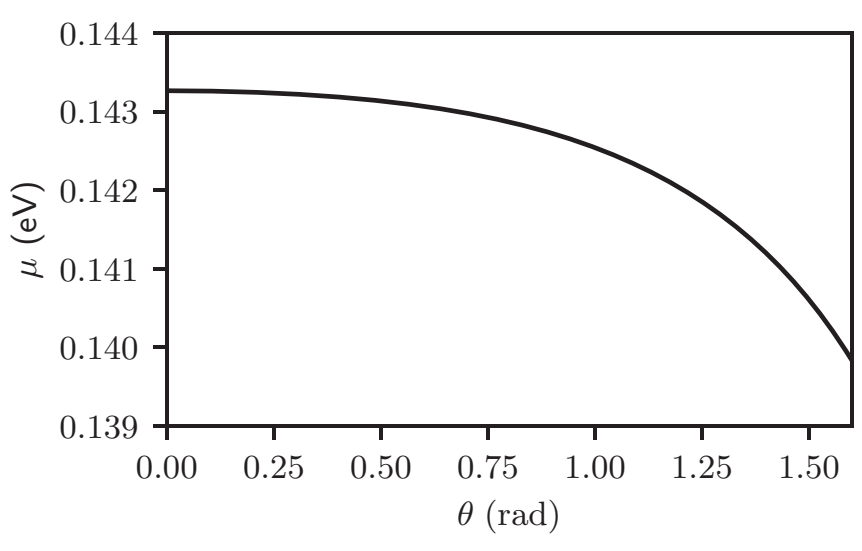

FIG. 8. Dependence of the chemical potential $\mu$ of an iron dimer on the angle $\theta$ between the two magnetic moments.

since parallel components do not contribute to the equation of motion (2.8).

\section{APPENDIX B: MATSUBARA SUMS}

For the evaluation of the exchange formulas, we have to calculate Matsubara sums over pairs of Green's functions, for example,

$$
X_{i j}^{\alpha \beta}=-\frac{\hbar^{2} \gamma^{2}}{4} \int_{\omega} \operatorname{Tr}\left\{\sigma_{i}^{\alpha} \mathcal{G}(i \omega) \sigma_{j}^{\beta} \mathcal{G}(i \omega)\right\} .
$$

We use the eigenbasis expansion of the Matsubara Green's function,

$$
\mathcal{G}(i \omega)=\sum_{n} \frac{|n\rangle\langle n|}{i \omega-\xi_{n}}
$$

where $\{|n\rangle\}$ are the single-electron eigenstates of the Hamiltonian with $\xi_{n}=\varepsilon_{n}-\mu$. Here, $\varepsilon_{n}$ is the energy of the state $|n\rangle$ and $\mu$ is the chemical potential that controls the occupation of the states, which we plot in Fig. 8 for the iron dimer. It is now straightforward to perform the summation over Matsubara frequencies,

$$
\int_{\omega} \frac{1}{\left(i \omega-\xi_{n}\right)\left(i \omega-\xi_{n^{\prime}}\right)}= \begin{cases}\frac{f\left(\xi_{n}\right)-f\left(\xi_{n^{\prime}}\right)}{\xi_{n}-\xi_{n^{\prime}}}, & \xi_{n} \neq \xi_{n^{\prime}} \\ f^{\prime}\left(\xi_{n}\right), & \xi_{n}=\xi_{n^{\prime}}\end{cases}
$$

where the Fermi function and its derivative are given by

$$
\begin{gathered}
f\left(\xi_{n}\right)=\frac{1}{e^{\beta \xi_{n}}+1}, \\
f^{\prime}\left(\xi_{n}\right)=\frac{-\beta e^{\beta \xi_{n}}}{\left(e^{\beta \xi_{n}}+1\right)^{2}}=\beta f\left(\xi_{n}\right)\left[f\left(\xi_{n}\right)-1\right],
\end{gathered}
$$

with the inverse temperature $\beta=1 /\left(k_{B} T\right)$.

The Matsubara formalism that we employ here introduces a temperature dependence. Since we consider only the zerotemperature limit, it is important to confirm the convergence of our calculations for $T \rightarrow 0$. This is demonstrated in Fig. 9 for the Heisenberg exchange of an iron dimer in the ferromagnetic ground state, which shows only a weak temperature dependence for $T<300 \mathrm{~K}$.

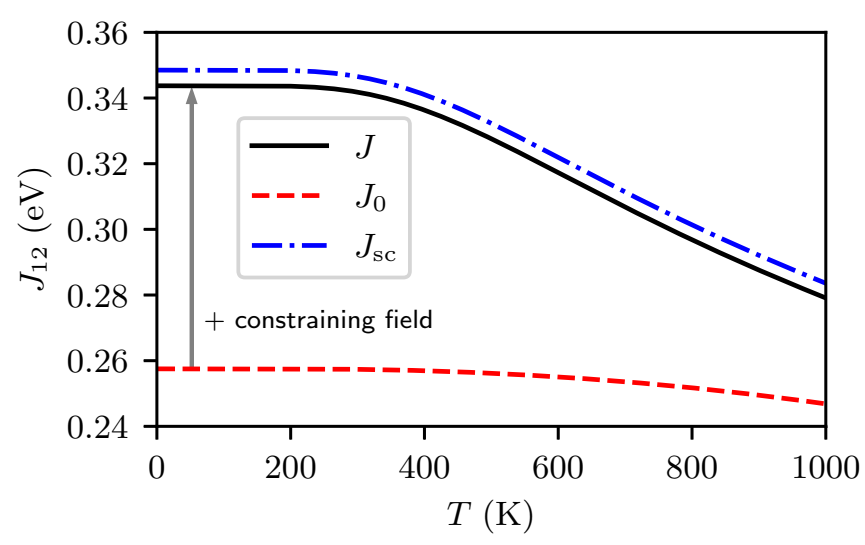

FIG. 9. Dependence of the Heisenberg exchange $J_{12}$ of a ferromagnetic iron dimer on the temperature parameter $T$ in the exchange formulas given by Eqs. (3.23), (3.34), and (3.33).

\section{APPENDIX C: SYMMETRY OF EXCHANGE CONSTANTS}

Exchange constants that are derived from the curvature of the energy,

$$
J_{i j}^{\alpha \beta}=-\frac{\partial^{2}\langle\hat{\mathcal{H}}\rangle}{\partial e_{i \alpha} \partial e_{j \beta}}
$$

are by definition symmetric with respect to the interchange $i \alpha \leftrightarrow j \beta$. While this fundamental symmetry is directly reflected in the exchange formula $J_{0, i j}^{\alpha \beta}$ in Eq. (3.34), it is not obvious that the derived formulas for $J_{i j}^{\alpha \beta}$ and $J_{\mathrm{sc}, i j}^{\alpha \beta}$ in Eqs. (3.23) and (3.33) fulfill this symmetry. We have therefore checked this symmetry numerically within our tight-binding calculations for an iron dimer. Our results in Fig. 10 show that $J_{\mathrm{sc}, i j}^{\alpha \beta}$ fulfills the symmetry exactly, whereas $J_{i j}^{\alpha \beta}$ breaks it for $\theta \neq 0$, although the asymmetry reaches only the order of $1 \%$. This asymmetry is not related to the asymmetry that can be introduced by the projection discussed in Appendix A because we have not used this projection here.

From the constraining field theorem [28], we obtain the following relation for the exchange constant $J_{i j}^{\alpha \beta}$ obtained

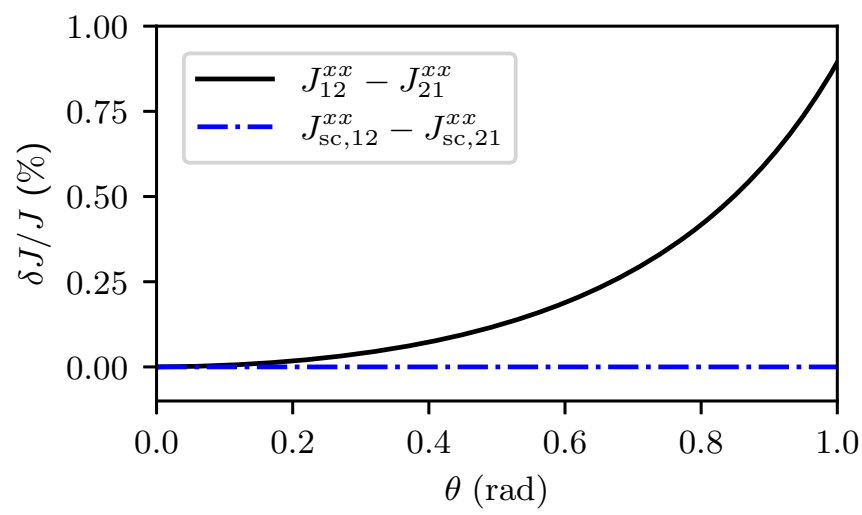

FIG. 10. Numerical check of the symmetry of the exchange constants $J_{i j}^{x x}$ and $J_{\mathrm{sc}, i j}^{x x}$ of an iron dimer as a function of the angle $\theta$ between the two magnetic moments. 
from the constraining field via Eq. (3.3):

$$
J_{i j}^{\alpha \beta}=-\frac{\partial^{2}\left\langle\hat{\mathcal{H}}_{\mathrm{tb}}\right\rangle}{\partial e_{i \alpha} \partial e_{j \beta}}+\frac{\partial}{\partial e_{j \beta}}\left\langle\frac{\partial \hat{\mathcal{H}}_{\mathrm{tb}}}{\partial e_{i \alpha}}\right\rangle .
$$

The fundamental tight-binding Hamiltonian $\hat{\mathcal{H}}_{\mathrm{tb}}$ as defined in Eq. (3.6) is independent of the moment directions $\left\{\mathbf{e}_{i}\right\}$, which implies that $J_{i j}^{\alpha \beta}$ is symmetric since the second term on the right-hand side of Eq. (C2) vanishes in that case. However, our numerical calculations are based on a mean-field tightbinding model where the electron-electron interactions are effectively included within a mean-field approximation via the Stoner term, given by Eq. (3.29). This Stoner term depends on the moment directions $\left\{\mathbf{e}_{i}\right\}$ and causes, in our calculations, the asymmetry of $J_{i j}^{\alpha \beta}$. The symmetry-breaking term is subtracted in the definition of $J_{\mathrm{sc}, i j}^{\alpha \beta}$, given by Eq. (3.28), such that

$$
J_{\mathrm{sc}, i j}^{\alpha \beta}=-\frac{\partial^{2}\left\langle\hat{\mathcal{H}}_{\mathrm{tb}}\right\rangle}{\partial e_{i \alpha} \partial e_{j \beta}},
$$

where we assume constant charges and moment lengths, i.e., $\nabla_{\mathbf{e}_{i}} \equiv \nabla_{\mathbf{e}_{i}}^{*}$, which is consistent with the approximations made to derive the exchange formulas in Sec. III. The quantity $\left\langle\hat{\mathcal{H}}_{\mathrm{t}}\right\rangle$ describes the band energy of the tight-binding model, while the total energy contains additional constant energy contributions that arise from the mean-field decoupling and are not included in $\hat{\mathcal{H}}_{\mathrm{tb}}$ here. The exchange constant $J_{i j}^{\alpha \beta}$ is derived from the constraining field, which corresponds to the gradient of the total energy [28], explaining the difference between $J_{i j}^{\alpha \beta}$ and $J_{\mathrm{sc}, i j}^{\alpha \beta}$. Without the mean-field approximation, this difference would not arise.
[1] M. Takahashi, Half-filled Hubbard model at low temperature, J. Phys. C: Solid State Phys. 10, 1289 (1977).

[2] A. H. MacDonald, S. M. Girvin, and D. Yoshioka, $\frac{t}{U}$ expansion for the Hubbard model, Phys. Rev. B 37, 9753 (1988).

[3] M. Hoffmann and S. Blügel, Systematic derivation of realistic spin models for beyond-heisenberg solids, Phys. Rev. B 101, 024418 (2020).

[4] J. Kübler, Theory of Itinerant Electron Magnetism (Oxford University Press, Oxford, 2000).

[5] R. Drautz and M. Fähnle, Spin-cluster expansion: Parametrization of the general adiabatic magnetic energy surface with $a b$ initio accuracy, Phys. Rev. B 69, 104404 (2004).

[6] R. Drautz and M. Fähnle, Parametrization of the magnetic energy at the atomic level, Phys. Rev. B 72, 212405 (2005).

[7] A. Antal, B. Lazarovits, L. Udvardi, L. Szunyogh, B. Újfalussy, and P. Weinberger, First-principles calculations of spin interactions and the magnetic ground states of Cr trimers on $\mathrm{Au}(111)$, Phys. Rev. B 77, 174429 (2008).

[8] S. Grytsiuk, J.-P. Hanke, M. Hoffmann, J. Bouaziz, O. Gomonay, G. Bihlmayer, S. Lounis, Y. Mokrousov, and S. Blügel, Topological-chiral magnetic interactions driven by emergent orbital magnetism, Nat. Commun. 11, 511 (2020).

[9] S. Brinker, M. dos Santos Dias, and S. Lounis, Prospecting chiral multisite interactions in prototypical magnetic systems, Phys. Rev. Res. 2, 033240 (2020).

[10] A. I. Liechtenstein, M. I. Katsnelson, and V. A. Gubanov, Exchange interactions and spin-wave stiffness in ferromagnetic metals, J. Phys. F 14, L125 (1984).

[11] A. I. Liechtenstein, M. I. Katsnelson, V. P. Antropov, and V. A. Gubanov, Local spin density functional approach to the theory of exchange interactions in ferromagnetic metals and alloys, $\mathrm{J}$. Magn. Magn. Mater. 67, 65 (1987).

[12] S. A. Turzhevskii, A. I. Likhtenstein, and M. I. Katsnelson, Degree of localization of magnetic moments and the nonHeisenberg nature of exchange interactions in metals and alloys, Sov. Phys. Solid State 32, 1138 (1990).

[13] A. Secchi, S. Brener, A. Lichtenstein, and M. Katsnelson, Non-equilibrium magnetic interactions in strongly correlated systems, Ann. Phys. (NY) 333, 221 (2013).
[14] A. Secchi, A. I. Lichtenstein, and M. I. Katsnelson, Nonequilibrium itinerant-electron magnetism: A time-dependent meanfield theory, Phys. Rev. B 94, 085153 (2016).

[15] V. Antropov, M. Katsnelson, and A. Liechtenstein, Exchange interactions in magnets, Physica B Condens. Matter 237-238, 336 (1997).

[16] V. Antropov, B. Harmon, and A. Smirnov, Aspects of spin dynamics and magnetic interactions, J. Magn. Magn. Mater. 200, 148 (1999).

[17] A. Szilva, M. Costa, A. Bergman, L. Szunyogh, L. Nordström, and O. Eriksson, Interatomic Exchange Interactions for FiniteTemperature Magnetism and Nonequilibrium Spin Dynamics, Phys. Rev. Lett. 111, 127204 (2013).

[18] A. Secchi, A. Lichtenstein, and M. Katsnelson, Magnetic interactions in strongly correlated systems: Spin and orbital contributions, Ann. Phys. (NY) 360, 61 (2015).

[19] A. Szilva, D. Thonig, P. F. Bessarab, Y. O. Kvashnin, D. C. M. Rodrigues, R. Cardias, M. Pereiro, L. Nordström, A. Bergman, A. B. Klautau, and O. Eriksson, Theory of noncollinear interactions beyond Heisenberg exchange: Applications to bcc Fe, Phys. Rev. B 96, 144413 (2017).

[20] R. Cardias, A. Szilva, M. M. Bezerra-Neto, M. S. Ribeiro, A. Bergman, Y. O. Kvashnin, J. Fransson, A. B. Klautau, O. Eriksson, and L. Nordström, First-principles DzyaloshinskiiMoriya interaction in a noncollinear framework, Sci. Rep. 10, 20339 (2020).

[21] R. Cardias, A. Bergman, A. Szilva, Y. O. Kvashnin, J. Fransson, A. B. Klautau, O. Eriksson, and L. Nordström, Dzyaloshinskii-Moriya interaction in absence of spin-orbit coupling, arXiv:2003.04680.

[22] M. dos Santos Dias, S. Brinker, A. Lászlóffy, B. Nyári, S. Blügel, L. Szunyogh, and S. Lounis, Proper and improper chiral magnetic interactions, Phys. Rev. B 103, L140408 (2021).

[23] P. Bruno, Exchange Interaction Parameters and Adiabatic SpinWave Spectra of Ferromagnets: A "Renormalized Magnetic Force Theorem”, Phys. Rev. Lett. 90, 087205 (2003).

[24] V. Antropov, The exchange coupling and spin waves in metallic magnets: Removal of the long-wave approximation, J. Magn. Magn. Mater. 262, L192 (2003). 
[25] M. I. Katsnelson and A. I. Lichtenstein, Magnetic susceptibility, exchange interactions and spin-wave spectra in the local spin density approximation, J. Phys. Condens. Matter 16, 7439 (2004).

[26] G. M. Stocks, B. Ujfalussy, X. Wang, D. M. C. Nicholson, W. A. Shelton, Y. Wang, A. Canning, and B. L. Györffy, Towards a constrained local moment model for first principles spin dynamics, Philos. Mag. B 78, 665 (1998).

[27] B. Ujfalussy, X.-D. Wang, D. M. C. Nicholson, W. A. Shelton, G. M. Stocks, Y. Wang, and B. L. Gyorffy, Constrained density functional theory for first principles spin dynamics, J. Appl. Phys. 85, 4824 (1999).

[28] S. Streib, V. Borisov, M. Pereiro, A. Bergman, E. Sjöqvist, A. Delin, O. Eriksson, and D. Thonig, Equation of motion and the constraining field in ab initio spin dynamics, Phys. Rev. B 102, 214407 (2020).

[29] R. C. A. de Almeida, C. Barreteau, P. Thibaudeau, and C. C. $\mathrm{Fu}$, Spin dynamics from a constrained magnetic tight-binding model, arXiv:2101.06121.

[30] I. V. Solovyev, Exchange interactions and magnetic force theorem, Phys. Rev. B 103, 104428 (2021).

[31] A. Jacobsson, G. Johansson, O. I. Gorbatov, M. Ležaić, B. Sanyal, S. Blügel, and C. Etz, Parameterisation of noncollinear energy landscapes in itinerant magnets, arXiv:1702.00599.

[32] S. V. Halilov, H. Eschrig, A. Y. Perlov, and P. M. Oppeneer, Adiabatic spin dynamics from spin-density-functional theory: Application to Fe, Co, and Ni, Phys. Rev. B 58, 293 (1998).

[33] W. Kohn and L. J. Sham, Self-consistent equations including exchange and correlation effects, Phys. Rev. 140, A1133 (1965).
[34] M. S. S. Brooks and B. Johansson, Exchange integral matrices and cohesive energies of transition metal atoms, J. Phys. F: Met. Phys. 13, L197 (1983).

[35] G. Autès, C. Barreteau, D. Spanjaard, and M.-C. Desjonquères, Magnetism of iron: From the bulk to the monatomic wire, J. Phys. Condens. Matter 18, 6785 (2006).

[36] T. Schena, Tight-binding treatment of complex magnetic structures in low-dimensional systems, Diploma thesis, TH Aachen, 2010.

[37] M. I. Katsnelson and A. I. Lichtenstein, First-principles calculations of magnetic interactions in correlated systems, Phys. Rev. B 61, 8906 (2000).

[38] T. Nomoto, T. Koretsune, and R. Arita, Local force method for the $a b$ initio tight-binding model: Effect of spin-dependent hopping on exchange interactions, Phys. Rev. B 102, 014444 (2020).

[39] Danny Thonig, computer code CAHMD, Classical atomistic Heisenberg magnetization dynamics. A computer program package for atomistic magnetization dynamics simulations (unpublished), danny.thonig@oru.se, https://cahmd.gitlab.io/ cahmdweb/.

[40] J. C. Slater and G. F. Koster, Simplified LCAO method for the periodic potential problem, Phys. Rev. 94, 1498 (1954).

[41] D. Thonig and J. Henk, Gilbert damping tensor within the breathing Fermi surface model: Anisotropy and nonlocality, New J. Phys 16, 013032 (2014).

[42] S. Mankovsky, S. Polesya, and H. Ebert, Exchange coupling constants at finite temperature, Phys. Rev. B 102, 134434 (2020).

[43] M. Malki and G. S. Uhrig, Topological magnetic excitations, Europhys. Lett. 132, 20003 (2020). 